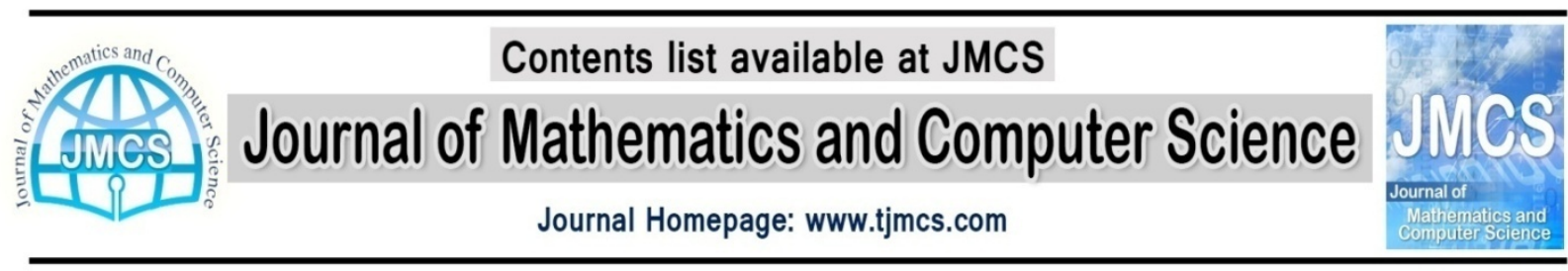

\title{
On the Amalgamated Duplication of an Unitary Normed Algebra Along an Ideal
}

\author{
Sara Dadras, Mahsa Jafarian \\ Department of Mathematics, Science and Research Branch, Islamic Azad University \\ Tehran, Iran \\ Sara_dadras@yahoo.com \\ mahsa.jafarian@yahoo.com
}

\section{Article history: \\ Received April 2013 \\ Accepted June 2013 \\ Available online June 2013}

\begin{abstract}
In this paper, based on an amalgamated duplication of a ring along an ideal, we will construct the amalgamated duplication of an unitary normed algebra $X$ along its proper ideal I (i.e. $X \bowtie I$ ). Then we will discus about its Banach conditions and abstract properties. Mainly we have proven that if $X$ ba a Banach algebra and $I$ be its closed ideal then $X \bowtie I$ will be Banach algebra too. As well, we have shown that its completion and ideals based on original normed algebra $X$. Finally some more aspects to expend these results for generalized normed algebras are given.
\end{abstract}

Keywords: Amalgamated duplication, Unitary normed Algebra, Banach Algebra,*-algebra.

\section{Introduction}

In recent years, there have been several investigations $[9,8,2,6]$ to find properties of the amalgamated duplication of a ring along an its proper ideal. This concept first was presented by [9]. More details, If $R$ is a commutative ring with identity and $I$ be a proper ideal of $R$, the following definition, which is as a sub-ring of $R \times R$

$$
R \bowtie I:=\{(x, y): x, y \in R \& y-x \in I\},
$$

was proposed by [9] as so called the amalgamated duplication of ring along an its ideal. It can be shown that the above structure is a extension for idealization which was originally introduced by [10] (See $[9,8]$ to more details).

Mathematical properties and applications of this type of extensions have been a motivation for some other papers (See e.g. $[1,3,11,5]$ ). 
Here, we have an attempt to extend such approach in operator theory. More details, we will define the $X \bowtie I$ for the unitary normed algebra $X$ and its proper ideal $I$, then we will show with some appropriate $d$ it forms a normed algebra as well. Then, we will discus about its some aspects like Banach conditions, abstract properties and closure and closed aspects. Mainly we will prove in section 3 that $X$ is an unitary Banach algebra if an only if $X \bowtie I$ is an unitary Banach algebra and $I$ is closed, as well as $X$ is Noetherian if and only if $X \bowtie I$ is Noetherian and $\overline{X \bowtie I}=\bar{X} \bowtie \bar{I}$.Moreover, to clear these aspect we will use some applicable illustrations, too. Roughly speaking, this paper gives most selected aspects of amalgamated duplication for normed algebras as well as carrying that construction in operator theory in both abstract and normed scopes. More specially, present paper organized as follows: In Sec 2, some basic concept about normed algebras as well as constructing amalgamated duplication of an unitary normed algebras along an ideal will given. Sec 3 will be devoted to main result about this new construction $X \bowtie I$ involved Banach conditions, abstract properties, closure and closed aspects. Some remarkable examples and corollaries are given in this section as well. Sec 4, discuses mainly about expending this construction for generalized normed algebra and expressing this results for normed rings.

\section{Preliminary}

In this section we will recall some basic notions from the operator theory, i.e.normed algebra and its aspects. The reader is referred to [7] for further details, and [3] for modern expositions and further developments.

Definition 2.1. $\quad$ A normed space $X$ that is simultaneously an algebra with respect to a product operation

$X \times X \rightarrow X$, say $(x, y) \mapsto x y$, such that $\|x y\| \leq\|x\|$. $\|y\|$ is called a normed algebra. If a normed algebra possesses an identity 1 such that $\|1\|=1$, then it is a unital normed algebra (or a normed algebra with identity).

Through this paper let $X$ be an unitary commutative normed algebra with norm $\|$. $\|$ and $I$ is a proper ideal of that. We can show the following set $X \bowtie I:=\{(x, y): x, y \in X \& y-x \in I\}$, with ordinary addition and multiplication is provide a new unitary algebra. More details, since $\left(x_{1}, y_{1}\right)+$ $\left(x_{2}, y_{2}\right)=\left(x_{1}+x_{2}, y_{1}+y_{2}\right)$ and $\alpha(x, y)=(\alpha x, \alpha y)$ for every $\left(x_{1}, y_{1}\right.$ and $\left(x_{2}, y_{2}\right)$ of $X \bowtie I$ and $\alpha$ in $F$ are belong to $X \bowtie I$, so it forms a subspace of $X \times X$. As well

$$
\left(x_{1}, y_{1}\right)\left(x_{2}, y_{2}\right)=\left(x_{1} x_{2}, y_{1} y_{2}\right)=y_{1}\left(y_{2}-x_{2}\right)+x_{2}\left(y_{1}-x_{1}\right)
$$

is belong to $X \bowtie I$. So we can conclude $X \bowtie I$ is a sub-algebra of $X \times X$ with identity $(1,1)$.

Now, we can define the real-valued function

$$
d: X \bowtie I \rightarrow R
$$

$$
(x, y) \mapsto\|x\|+\|y\|,
$$

where $\|$.$\| is norm of X$. It is easy to check that $d$ is defined a norm on $X \bowtie I$.Moreover, we have

$$
\begin{aligned}
d\left(\left(x_{1}, y_{1}\right)\left(x_{2}, y_{2}\right)\right. & =d\left(\left(x_{1} x_{2}, y_{1} y_{2}\right)\right)=\left\|x_{1} x_{2}\right\|+\left\|y_{1} y_{2}\right\| \\
\leq & \left\|x_{1}\right\|+\left\|x_{2}\right\|+\left\|y_{1}\right\|+\left\|y_{2}\right\| \\
= & d\left(\left(x_{1}, y_{1}\right)\right)+d\left(\left(x_{2}, y_{2}\right)\right)
\end{aligned}
$$

So, $X \bowtie I$ with $d$ forms an normed algebra which can be named as amalgamated duplication of unitary noremd algebra along an ideal.

Remark 2.2. As we can see through norm (2.1) $X \bowtie I$ is not unitary normed algebra. Actually we find $d(1,1)=\|1\|+\|1\|=2$.

Remark 2.3. We can define $d$ in other ways. For example as form $d^{\prime}((x, y))=\|x\|$ or $d^{\prime \prime}((x, x+$ $i))=|| x \mid+\|i\|$. As we can show $X \bowtie I$ with $d^{\prime}$ is unitary semi-noremd algebra and with $d^{\prime \prime}$ is unitary semi-normed algebra, too. But in this paper we have chosen $d$ as form (2.1) in order to have a normed algebra.

To finish this section we point out that every element of $L(X)$ (linear space of all linear transformations on $X)$ induces an element for $L(X \bowtie I)$. More details, Consider the following maps

$$
\pi: X \bowtie I \rightarrow X \&(x, x+i) \mapsto x,
$$




$$
\pi^{\prime}: X \rightarrow X \bowtie I \& x \mapsto(x, x) .
$$

It is easy to check that the above maps are linear transformations. Based on them,consider map $\psi$ :

$$
\psi: L(X) \rightarrow L(X \bowtie I) \& T \mapsto \pi^{\prime} \circ T \text { o } \pi
$$

Since $\psi$ is a composition of linear transforation, so it is linear and well-defined too.Let $T^{\prime} \in$ ker $\psi$. So, we have $\pi^{\prime} o T^{\prime} o \pi((x, x+i))=0$ for every $(x, x+i) \in X \bowtie I$.

The last equality can be expressed $\pi^{\prime}\left(\mathrm{T}^{\prime} o \pi((\mathrm{x}, \mathrm{x}+\mathrm{i}))\right)=0$. Since $\pi^{\prime}$ is injective,so we can conclude $T^{\prime} o \pi((x, x+i))=0$, i.e $T^{\prime}(x)=0$ for every $x \in X$. As a result: $T^{\prime} \equiv 0$ and hence $\psi$ is injective.

Remark 2.4. The above result similarly can be translated to the $L(X, F)$ i.e. dual space on $X$.

In the next section, we will have an attempt to present more results as well as some applicable illustration about this new construction $X \bowtie I$.

\section{Main title}

Through this section consider $X$ as an arbitrary unitary normed algebra with proper ideal $I$.

First, we want to investigate that whether $X \bowtie I$ is Banach algebra for the Banach algebra $X$. For this, we will begin with following elementary definitions.

Defnition 3.1. A Banach algebra is a normed algebra that is complete as a normed space.

Defnition 3.2. A metric space $X$ is complete if every Cauchy sequence in $X$ is a convergent sequence in $X$.

Next result mainly is investigated Banach conditions for $X \bowtie I$ :

Theorem 3.3. $X$ is an unitary Banach algebra if an only if $X \bowtie I$ is an unitary Banach algebra and $I$ is closed.

Proof. Let $\left\{\left(x_{n}, \mathrm{y}_{\mathrm{n}}\right)\right\}$ be a Cauchy sequence in $\mathrm{X} \bowtie \mathrm{I}$ with $\mathrm{X}$ as a Banach algebra.Since $\mathrm{X}$ is Banach, So sequence $\left\{x_{n}\right\}$ and $\left\{\mathrm{y}_{\mathrm{n}}\right\}$ are convergent. As a result sequence $\left\{\left(x_{n}, \mathrm{y}_{\mathrm{n}}\right)\right\}$ is convergent (say to $(\mathrm{x}, \mathrm{y})$ ). Moreover, if we consider new subtraction sequence $\left\{\mathrm{y}_{\mathrm{n}}-\mathrm{x}_{\mathrm{n}}\right\}$ trivially it converges to $\mathrm{y}-\mathrm{x}$. Since I is a closed ideal, we can conclude $\mathrm{y}-\mathrm{x}$ is in I and as a result $(\mathrm{x}, \mathrm{y})$ is lying in $\mathrm{X} \bowtie \mathrm{I}$. Hence, $\mathrm{X} \bowtie \mathrm{I}$ is a

Banach algebra.

Conversely, Let $\mathrm{X} \bowtie \mathrm{I}$ be Banach algebra and $\left\{\mathrm{x}_{\mathrm{n}}\right\}$ be a cauchy sequence in X.So, sequence $\left\{\left(x_{n}, \mathrm{x}_{\mathrm{n}}\right)\right\}$ is a Cauchy in $X \bowtie I$ and it is convergent (say $(x, x)$ ). As a result $\{x n\}$ is convergent and hence $X$ is Banach as well.

We know that any Banach algebra (whether it has an identity element or not) can be embedded isometrically into a unitary Banach algebra $X_{\epsilon}$ so as to form a closed ideal of $X_{\epsilon}$. So, the two following results are hold:

Corollary 3.4. If $X$ be a Banach algebra and $I$ be its proper closed ideal, then $X \bowtie I$ can be embedded in an unique unitary Banach algebra.

Corollary 3.5. If $X$ be a Banach algebra whether it has an identity element or not, then $X_{\epsilon} \bowtie X$ is unitary Banach algebra.

Example 3.6. Let $X$ and $Y$ as Banach algebras. In [6] was shown that $B(X, Y)$ (the set of all bounded linear transformation from $X$ to $Y$ )is a Banach algebra and $B_{\infty}(X, Y)$ is its closed ideal, so by above theorem the amalgamated duplication $B[X, Y] \bowtie B_{\infty}(X, Y)$ forms a Banach algebra as well.

Next proposition provide more Banach amalgamated algebras:

Proposition 3.7. If $X$ be a Banach algebra and $I$ be a maximal regular ideal, then $X \bowtie I$ is Banach algebra.

Proof. In [13] was proven that maximal regular ideals are closed. So using that fact the proof is clear.

One of the most important properties that must be considered for $X \bowtie I$ is form of its closure. The closure of any normed algebra $X$ is defined as a set consists of all points in $X$ plus the limit points of $X$ and that shown by $\bar{X}$ The following preposition mainly is investigated closure of $X \bowtie I$ using closure of $X$ and $I$.

Proposition 3.8. We have: 


$$
\overline{X \bowtie I}=\bar{X} \bowtie \bar{I}
$$

Proof. First we must note that since $I$ is a proper ideal for $X$ we can conclude that $\bar{I}$ is a proper ideal for $\bar{X}$, and the definition of $\bar{X} \bowtie \bar{I}$ will be well defined.

Consider $(x, x+i) \in \overline{X \bowtie I}$, so there is sequence $\left(x_{n}, x_{n}+i_{n}\right)$ in $X \bowtie I$ such that $\left(x_{n}, x_{n}+i_{n}\right) \rightarrow$ $(x, x+i)$. Hence we can conclude

$$
x_{n} \rightarrow x \text { and } i_{n} \rightarrow i
$$

so $x$ is belong to $\bar{X}$ and $i$ is belong to $\bar{I}$. As a result $(x, x+i)$ is in $\bar{X} \bowtie \bar{I}$.

Conversely, consider $(x, y)$ in $\bar{X} \bowtie \bar{I}$. So, we have a sequence like $\left(x_{n}, y_{n}\right)$ such that $\left(x_{n}, y_{n}\right) \rightarrow$ $(x, y)$ and $x-y$ is belong to $\bar{I}$. Since $x_{n}$ and $y_{n}$ is belong to $X$ and $x_{n}-y_{n}$ is in $I$, so $x_{n}, y_{n}$ are belong to $X \bowtie I$ and as a result $(x, y)$ is belong to $\overline{X \bowtie I}$.

The above theorem gives us being closed conditions for $X \bowtie I$ :

Corollary 3.9. $X \bowtie \mathrm{I}$ is closed if and only if $X$ and $I$ are closed.

Proof. If $X$ and $I$ were closed, so we have $\bar{X} \bowtie \bar{I}=X \bowtie I$, and then from above theorem we can conclude $\overline{X \bowtie I}=X \bowtie I$

Conversely, if $X \bowtie I$ be closed, then we have $\bar{X} \bowtie \bar{I}=X \bowtie I$. So $\bar{X}=X$ and $\bar{I}=I$ and as a result $X$ and $I$ are closed.

The concept of *-algebra plays an important role in algebra theory [12]. *-algebra is an algebra equipped with an algebra involution and An algebra involution on an algebra $X$ is an involution $x \mapsto$ $x^{*}$ on $X$ such that for all $\lambda, \mu$ in $C$ and all $x, y$ in $X$, we have

$$
\begin{aligned}
(\lambda x+\mu y)^{*} & =\bar{\lambda} x^{*}+\bar{\mu} y^{*} \\
(x y)^{*} & =y^{*} x^{*} .
\end{aligned}
$$

The following preposition is devoted to relations between involutions of $X$ and $X \bowtie I$, and to condition of constructing $X \bowtie I$ as the *-algebra using involution of $X$.

Proposition 3.10. Let $X$ be the *-algebra with involution $x \rightarrow x^{*}$ and $I$ be its proper ideal, then we can construct involution for $X \bowtie I$, if we have $I^{*} \subseteq I$.

Proof. Consider the following map on $X \bowtie I$ :

$$
\alpha: \quad \begin{array}{cl}
X \bowtie I & \rightarrow X \bowtie I \\
(x, x+i) & \mapsto\left(x^{*}, x^{*}+i^{*}\right) .
\end{array}
$$

since $I^{*} \subset I$ so the above map is well defined. For conditions (3.2) we get

$\alpha(\lambda(x, x+i)+\mu(y, y+J))=\alpha((\lambda x+\mu y, \lambda x+\mu y+\lambda i+\mu j))$

$$
\begin{aligned}
& =\left((\lambda x+\mu y)^{*},(\lambda x+\mu y)^{*}+(\lambda i+\mu j)^{*}\right) \\
& =\left(\bar{\lambda} x^{*}+\bar{\mu} y^{*}, \bar{\lambda} x^{*}+\bar{\mu} y^{*}+\bar{\lambda} i^{*}+\bar{\mu} j^{*}\right) \\
& =\bar{\lambda}\left(x^{*}, x^{*}+i^{*}\right)+\bar{\mu}\left(y^{*}, y^{*}+j^{*}\right) \\
& =\bar{\lambda} \alpha((x, x+i))+\bar{\mu} \alpha((y, y+j)),
\end{aligned}
$$

and

$$
\begin{aligned}
\alpha((x, x+i)(y, y+j)) & =\alpha((x y, x y+i j))=\left((x y)^{*}+(x y)^{*}+(i j)^{*}\right) \\
& =\left(y^{*} x^{*}, y^{*} x^{*}+j^{*} i^{*}\right)=\left(\left(y^{*}, y^{*}+j^{*}\right)\right)\left(\left(x^{*}, x^{*}+i^{*}\right)\right) \\
& =\alpha((y, y+j)) \alpha((x, x+i)) .
\end{aligned}
$$

Hence the map (3.3) is an involution on $X \bowtie I$ and as a result $X \bowtie I$ will be an $*$-algebra.

Large of important results for $X \bowtie I$ can be inherited from detected properties of $R \bowtie I$ (i.e. amalgamated duplication of a ring along an its ideal) which were established in $[2,4,8,9]$. In continuation some of them are given.

Theorem 3.11. $X$ is Noetherian if and only if $X \bowtie I$ is Noetherian.

Proof. It is not difficult to see that, if $\pi_{i}(i=1,2)$ are the projections of $X \times X$ on $X$, then $\pi_{i}(X \bowtie I)=X$; hence, if $O_{i}=\operatorname{ker}\left(\pi_{i} \mid X \bowtie I\right)$, then $X \cong(X \bowtie I) / O_{i}$.

In fact, if $X$ is Noetherian, then also $X \times X$ is Noetherian, hence $X \bowtie I$ is finitely generated as $X$ module and so is Noetherian; conversely, if $X \bowtie I$ is Noetherian, then $X \cong(X \bowtie I) / O_{i}$ is Noetherian(To more details see [8]). 
Theorem 3.12. Let $P$ be a prime ideal of $X$ and set:

$$
\begin{gathered}
P_{0}=\{(p, p+i) \mid p \in \mathrm{P}, i \in I \cap \mathrm{P}\}, \\
P_{1}=\{(p, p+i) \mid p \in \mathrm{P}, i \in I\}, \\
P_{2}=\{(p+i, p) \mid p \in \mathrm{P}, i \in I\} .
\end{gathered}
$$

- If $I \subseteq P$, then $P_{0}=P_{1}=P_{2}$ is a prime ideal of $X \bowtie I$ and it is the unique prime ideal of $X \bowtie I$ lying over $P$.

- If $I \nsubseteq P$, then $P_{1} \neq P_{2}, P_{1} \cap P_{2}=P_{0}$ and $P_{1}$ and $P_{2}$ are the only prime ideals of $X \bowtie I$ lying over $P$.

Corollary 3.13. If $X$ is local, with maximal ideal $m$, then $X \bowtie I$ is local with maximal ideal

$$
m_{0}=\{(x, x+i) \mid x \in m, i \in I\} .
$$

\section{More discussions}

As before, in this section we have an attempt to extend our new construction to generated normed algebras as well as express that for noremd rings. For this we will begin with two essential definitions: Definition 4.1. A generalized normed algebra $X$ is an abstract algebra with family of norms which satisfy in the following condition:

$$
\|x y\| \leq\|x\| .\|y\|,
$$

for every $\mathrm{x}$ and $y$ in $X$ and for every norm $\|$.$\| in that family.$

Definition 4.2. A normed ring is, a linear algebra $R$ over the complex numbers or the real numbers with a norm having, besides the usual properties of a norm, also the "ring" property

for every $x$ and $y$ in $R$.

$$
\|x y\| \leq\|x\| .\|y\|,
$$

Using the method which we allied in Sec.2, we can equip an amalgamated duplication structure to both normed ring and generalized normed algebra. As well all established statements can be extended to both normed ring and Generalized normed algebra.

For instance, we have:

Theorem 4.3. Let $X$ be an Generalized unitary normed algebra. Then $X$ is Banach if an only if $X \bowtie I$ is an Generalized unitary Banach algebra and $I$ is closed.

and

Proposition 4.4. Let $R$ be a normed ring, then we have:

$$
\overline{\mathrm{R} \bowtie \mathrm{I}}=\bar{R} \bowtie \bar{I}
$$

Without any doubt, These extensions makes the work of studying the amalgamated duplication of an unitary normed algebra more expediently.

\section{Conclusions}

In this work, starting with an unitary normed algebra $X$ and its proper ideal $I$, we have introduced new construction $X \bowtie I$ which can be named amalgamated duplication of an unitary normed algebra along an its ideal. And we proved that essential and sufficient conditions for Banach and abstract proprieties of it like ideals, primes and Noetherian. As well, some aspect and generalization of that to $C^{*}$-algebras and normed rings have expressed. These make the work of studying the amalgamated duplication more expediently.

\section{References}

[1] Akram B. Attar, Edge Extension Of Graphs And Digraphs, The Journal of Mathematics and Computer Science Vol .3 No.1 (2011) 1-10.

[2] A. Bagheri, M. Salimi, E. Tavasoli, S. Yassemi, A construction of Quasi-Gorenstein rings,Journal of Algebra and Its Applications Vol. 11, No. 1 (2012) 1250013 : 1-9. 
[3] Ball, J.A., Dym, H., Kaashoek, M.A., Langer, H., Tretter, C., The series in Operator Theory:Advances and Applications., springer.

[4] E. Tavasoli, M. Salimi, A. Tehranian, Amalagamted duplication of some special rings, Bull.Korean Math. Soc. 49 (2012), No. 5, pp. 989-996.

[5] H. Ananthnarayan, L.L. Avramov, W. Frank Moore, Conected sums of gorenstein local rings,arXiv:1005.1304v2 [math.AC] 10 Feb 2011.

[6] J. Shapiro, On a construction of Gorenstein rings proposed by M. DAnna, Journal of Algebra 323 (2010) 1155-1158.

[7] Kubrusly, Carlos S., Elements of operator theory, Birkh $\square$ auser Boston, co Springer-Verlag New York (1947).

[8] M. D'Anna, A construction of Gorenstein rings, Journal of Algebra 306 (2006) 507-519.

[9] M. D'Anna, M. Fontana, An amalgamated duplication of a ring along an ideal,Journal of Algebra and Its Applications Vol. 6, No. 3 (2007) 443-459.

[10] M. Nagata, Local Rings, Interscience, New York, 1962.

[11] M. Salimi, E. Tavasoli, S. Yassemi, The amalgamated duplication of a ring along a semidu-alizing ideal, Rend. Sem. Mat. Univ. Padova Manoscritto in corso di stampa, (2012).

[12] M.Thill Introduction to Normed _-Algebras and their Representations, 7th ed.arXiv:1011.1558v1 [math.OA] 6 Nov 2010.

[13] Y.Khomskii Banach Algebras, Bachelor Thesis, 2005. 